\title{
Lenvatinib vs. palliative therapy for stage IVC anaplastic thyroid cancer
}

\author{
HIROYUKI IWASAKI ${ }^{1}$, SOJI TODA ${ }^{1}$, NOBUYASU SUGANUMA ${ }^{1}$, DAISUKE MURAYAMA ${ }^{1}$, \\ HIROTAKA NAKAYAMA $^{2}$ and KATSUHIKO MASUDO ${ }^{3}$ \\ ${ }^{1}$ Department of Breast and Endocrine Surgery, Kanagawa Cancer Center, Yokohama, Kanagawa 241-8515; \\ ${ }^{2}$ Department of Surgical Treatment, Yokohama City University Hospital, Yokohama, Kanagawa 236-0004; \\ ${ }^{3}$ Department of Breast and Thyroid Surgery, Yokohama City University Medical Center, \\ Yokohama, Kanagawa 232-0024, Japan
}

Received July 19, 2019; Accepted November 20, 2019

DOI: $10.3892 / \mathrm{mco} .2019 .1964$

\begin{abstract}
Anaplastic thyroid cancer (ATC) is an orphan disease with extremely poor prognosis. In particular, unresectable stage IVC ATC is extremely difficult to treat and is associated with a survival of only a few months, even when treated with irradiation and/or chemotherapy. In 2015, lenvatinib was approved for the treatment of ATC in Japan. The aim of the present study was to evaluate the efficacy of lenvatinib for stage IVC ATC. A total of 32 patients with pathologically confirmed stage IVC ATC who were treated at the Kanagawa Cancer Center between 2011 and 2018 were included in the present study, of whom 16 patients were treated with lenvatinib (L group). The remaining 16 patients received palliative therapy (P group), of whom 7 were treated with weekly paclitaxel, 2 received external radiation for tumor reduction 5 days per week until treatment completion, and 2 underwent tracheostomy to avoid the risk of asphyxiation. The survival curves of both groups were analyzed using the log-rank test. The median overall survival time of the $\mathrm{L}$ and $\mathrm{P}$ groups was 4.2 and 2.0 months, respectively. A significant survival benefit was observed in the $\mathrm{L}$ group compared with that in the $\mathrm{P}$ group $(\mathrm{P}=0.00298)$. A reduction in tumor size by $\geq 30 \%$ (clinical partial response) within 1 month after treatment was observed in 5 patients $(31.3 \%)$ in the $\mathrm{L}$ group and in no patients in the $\mathrm{P}$ group. Therefore, lenvatinib treatment yielded a median
\end{abstract}

Correspondence to: Dr Hiroyuki Iwasaki, Department of Breast and Endocrine Surgery, Kanagawa Cancer Center, 2-3-2 Nakao Asahi-ku, Yokohama, Kanagawa 241-8515, Japan

E-mail: iwasaki.h@kcch.jp

Abbreviations: ATC, anaplastic thyroid cancer; BSC, best supportive care; DTC, differentiated thyroid cancer; PTX, paclitaxel; AE, adverse event; OS, overall survival; CI, confidence interval; CT, computed tomography; PTC, papillary thyroid carcinoma; FTC, follicular thyroid carcinoma; PD-1, programmed death-1

Key words: anaplastic thyroid cancer, lenvatinib, overall survival survival benefit of $\sim 2$ months compared with palliative therapy in stage IVC ATC. However, although a reduction in tumor size by $\geq 30 \%$ was confirmed in 5 patients who received lenvatinib treatment, 2 of those patients succumbed to massive necrosis and bleeding. These results suggest that an appropriate lenvatinib dose reduction is necessary.

\section{Introduction}

Anaplastic thyroid cancer (ATC) is a rare and lethal type of thyroid cancer. The survival rate of patients with ATC has not changed markedly over the past 20 years (1). Although clinical trials (randomized and non-randomized) have been conducted (2-4), including one with a large cohort of 205 patients, the considerable improvement in the understanding of the pathogenesis and genetics of ATC has not notably improved patient outcome (5). According to the American Thyroid Association guidelines, once a patient is diagnosed with stage IVC ATC, either systemic treatment and/or palliative treatment, including best supportive care (BSC), should be immediately implemented (6). Systemic therapy trials are time-consuming, and disease progression is quicker than the trial entry process prior to treatment initiation; therefore, the patient may not have sufficient time for treatment (7). In 2015, the Japanese Ministry of Health, Labor and Welfare approved the clinical use of lenvatinib for the treatment of patients with ATC and differentiated thyroid cancer (DTC). Therefore, lenvatinib may be used for the prompt systemic treatment of stage IVC ATC (8). Conversely, when patients treated with weekly paclitaxel (PTX), palliative radiation, or tracheostomy for local control manifest disease progression, BSC is administered to prolong survival. To the best of our knowledge, no studies to date have compared such cases and their prognoses, or have reported basic data of such cases. Although ATC is hypothesized to evolve from DTC, no report has yet proven that ATC originates from DTC $(9,10)$. The aim of the present study was to examine whether the clinical course of ATC was different from that determined using the presence and the shape of calcifications observed on computed tomography (CT) scans at the time of diagnosis. 
Table I. Patient characteristics.

\begin{tabular}{|c|c|c|c|}
\hline Variables & L group & P group & P-value \\
\hline $\mathrm{N}$ & 16 & 16 & \\
\hline Age, years [mean (range)] & $73.00(47.00-89.00)$ & $72.50(61.00-86.00)$ & 0.624 \\
\hline $\operatorname{Sex}, \mathrm{n}(\%)$ & & & 0.722 \\
\hline Female & $8(50.0)$ & $10(62.5)$ & \\
\hline Male & $8(50.0)$ & $6(37.5)$ & \\
\hline Body weight, kg [mean (range)] & $57.85(41.70-88.00)$ & $45.80(36.30-76.00)$ & 0.136 \\
\hline Lung metastasis, $\mathrm{n}(\%)$ & $16(100.0)$ & $14(87.5)$ & 0.484 \\
\hline Other metastasis, $\mathrm{n}(\%)$ & $4(25.0)$ & $4(25.0)$ & 1.000 \\
\hline Calcification, n (\%) & $10(71.4)$ & $8(57.1)$ & 0.695 \\
\hline Maximum diameter, mm [mean (range)] & $47.00(24.00-65.00)$ & $59.25(30.00-91.50)$ & 0.138 \\
\hline FT3, pg/ml [mean (range)] & $2.03(1.00-3.19)$ & $2.22(1.31-3.06)$ & 0.57 \\
\hline FT4, ng/dl [mean (range)] & $1.18(0.64-1.80)$ & $1.16(0.68-1.49)$ & 0.593 \\
\hline TSH, mIU/l [mean (range)] & $1.64(0.01-31.99)$ & $2.96(0.09-33.03)$ & 0.593 \\
\hline Hypothyroidism $^{\mathrm{b}}, \mathrm{n}(\%)$ & $14(87.5)$ & $4(25.0)$ & $0.001^{\mathrm{a}}$ \\
\hline
\end{tabular}

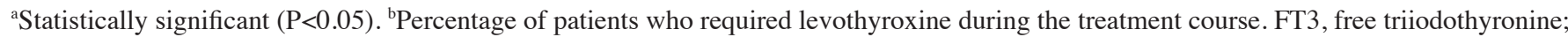
FT4, free thyroxine; TSH, thyroid-stimulating hormone; L, lenvatinib; P, paclitaxel.

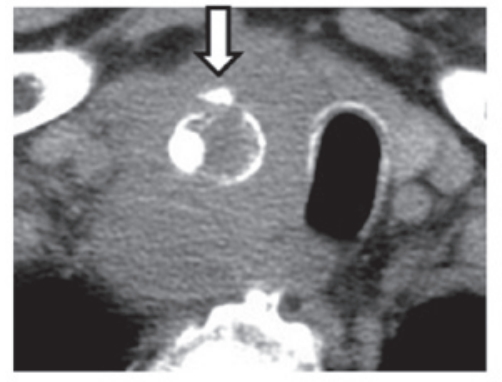

Egg-shell type calcification

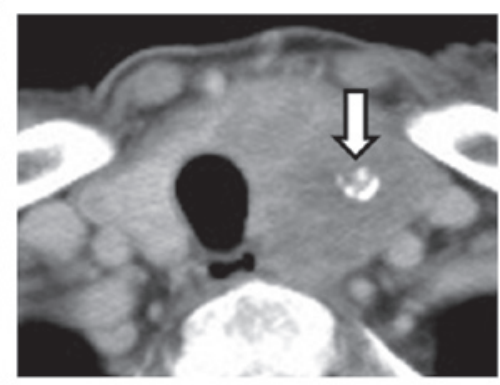

Lump calcification

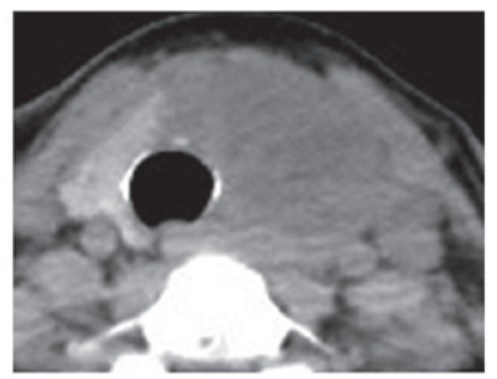

Without calcification

Figure 1. Calcification in the primary tumor. The tumor characteristics at diagnosis were classified into eggshell, lump and no calcification. White arrows, calcifications.

\section{Materials and methods}

Patients. A total of 32 patients with pathologically confirmed stage IVC ATC who were treated at the Kanagawa Cancer Center (Yokohama, China) between January 2011 and April 2019 were included in the present study. A total of 16 patients each were included in the lenvatinib $(\mathrm{L})$ and palliative $(\mathrm{P})$ groups. In the $\mathrm{P}$ group, 7 patients were treated with $80 \mathrm{mg} / \mathrm{m}^{2}$ PTX weekly, 2 underwent palliative irradiation, and 2 were subjected to tracheostomy for local control. Patients treated with PTX were included in the P group due to insufficient PTX dose for the evaluation of therapeutic efficacy. Patients in the L group were treated with lenvatinib alone. The background characteristics of patients in both groups are shown in Table I. Histopathological diagnosis was confirmed using core needle biopsy or open biopsy. Post-treatment disease progression and tumor diameter were assessed using CT scans every 4 weeks. Blood tests were conducted to determine thyroid function and the presence of adverse events (AEs). In the L group, the initial dose of lenvatinib was $24 \mathrm{mg}$ for 12 patients, $14 \mathrm{mg}$ for 3 patients, and $10 \mathrm{mg}$ for 1 patient. A total of 4 patients were administered a reduced dose due to poor performance status, malnutrition, and old age (>80 years). The survival curves of both groups were analyzed using the log-rank test. Tumor characteristics were classified into three subgroups according to the calcification patterns at the time of diagnosis as follows: Eggshell calcification, lump calcification, and no calcification (Fig. 1). Based on this classification, the results of lenvatinib treatment in cases with and without calcification were compared.

The Cancer Board of the Kanagawa Cancer Center approved lenvatinib treatment for ATC. The study protocol was approved by the Institutional Review Board of the Kanagawa Cancer Center (no. 49,2016).

Statistical analysis. The overall survival (OS) was calculated with the Kaplan-Meier method using SPSS software, v.24 (IBM Corp.). The Kaplan-Meier estimator on the SPSS software was 
Table II. Comparison of survival data between the L and P groups.

\begin{tabular}{lccc}
\hline Groups & $\mathrm{N}$ & 3-month survival rate (95\% CI) & Median survival [months (95\% CI)] \\
\hline $\mathrm{L}$ & 16 & $0.600(0.318-0.797)$ & $4.2(2.3-5.5)$ \\
$\mathrm{P}$ & 16 & $0.211(0.053-0.440)$ & $2.0(0.8-3.0)$
\end{tabular}

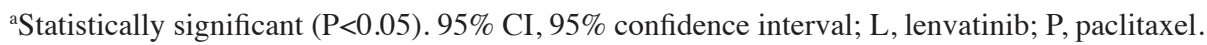

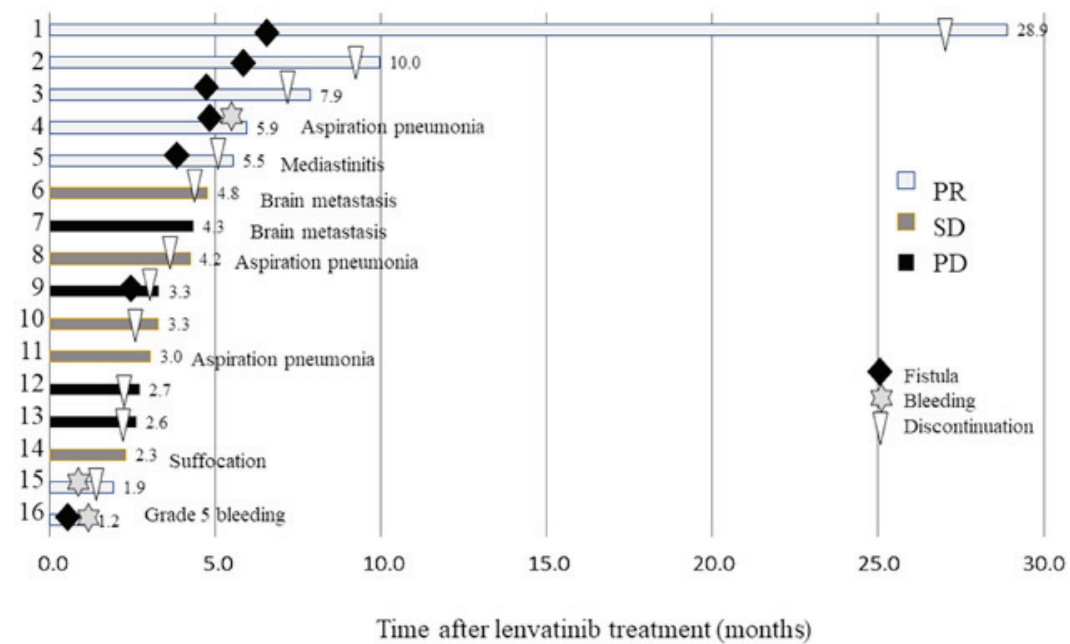

Figure 2. Treatment progression in 16 patients with ATC treated only with lenvatinib. Gray box, PR; dark gray box, SD; bracket box, PD; black diamonds, skin fistula; gray stars, massive bleeding; triangles, treatment discontinuation. The number to the right of the box indicates patient survival in months, and the non-disease related cause of death appears next to it. ATC, anaplastic thyroid cancer; PR, partial response; SD, stable disease; PD, progressive disease.

used to calculate OS and apply the log-rank test. $\mathrm{P}<0.05$ was considered to indicate statistically significant differences. The OS of the L and P groups was validated using the log-rank test. The median values between the two groups were compared using Man-Whitney $U$ test with statistical significance set at $\mathrm{P}<0.05$. The statistical analyses were performed using EZR (Saitama Medical Center, Jichi Medical University, Saitama, Japan), a graphical user interface for R (The R Foundation for Statistical Computing).

\section{Results}

Treatment results. In L group, the median duration of lenvatinib administration was 107.0 days (range, 30-837 days) (Fig. 2). There were 7 cases $(43.8 \%)$ of fistula formation (patients 1-5, 9 and 16), 2 cases (12.5\%) of bleeding (patients 4 and 16), and 1 case $(6.3 \%)$ of mediastinitis (patient 5$)$. There were 3 treatment-related deaths: Patient 16 succumbed to hemorrhage from a fistula, patient 4 developed aspiration pneumonia from a fistula, and patient 5 developed mediastinitis from a fistula. The remaining 13 deaths were considered as disease-specific, and some of the patients had other complications: 2 had brain metastases, 2 had aspiration pneumonia, and 1 developed suffocation. Patient 15 discontinued lenvatinib treatment due to grade 4 digestive tract bleeding. Other grade $\geq 3$ or AEs required dose reduction or discontinuation. None of the patients in the present study had undergone total thyroidectomy for volume reduction. All patients were diagnosed with stage IVC ATC and had no history of thyroid surgery. Necrosis of

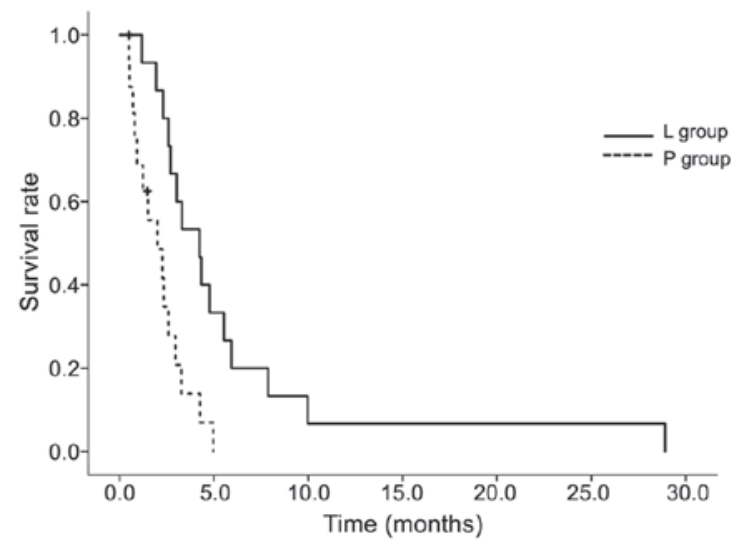

Figure 3. OS curves. Kaplan-Meier estimator (SPSS software)-derived survival curves indicate a median OS duration of 4.2 months in the L group and 2.0 months in the $\mathrm{P}$ group. A significant difference in OS was observed between the two groups $(\mathrm{P}=0.00298)$. OS, overall survival; $\mathrm{L}$, lenvatinib; $\mathrm{P}$, paclitaxel.

the tumor as well as of the normal thyroid gland by lenvatinib in the L group may have caused hypothyroidism.

In the P group, 7 patients (43.8\%) were treated with weekly PTX $\left(80 \mathrm{mg} / \mathrm{m}^{2}\right)$ as previously reported (11), but PTX was only administered 1-7 times. All patients in the P group had progressive disease and the treatment was discontinued. As shown in Table II and Fig. 3, a significantly different median survival benefit was observed between the $\mathrm{L}$ and $\mathrm{P}$ groups at 4.2 and 2 months $(\mathrm{P}=0.00298)$, respectively. 


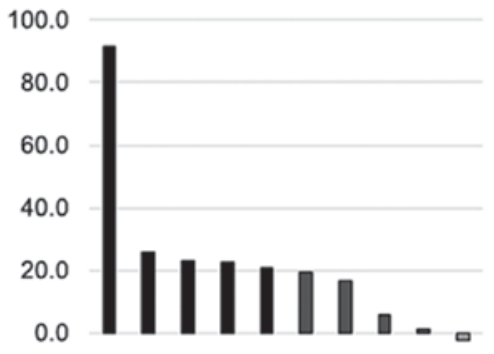

$-20.0$

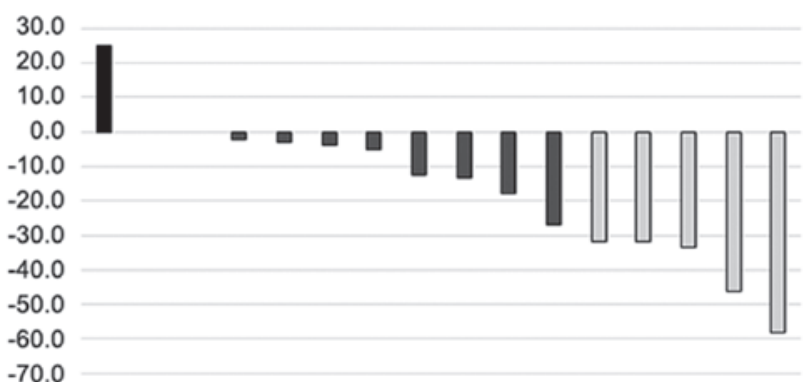

L group

Figure 4. Changes in tumor diameter in first 4 weeks after treatment. A tumor size change of $<-30 \%,-30 \%$ to $+20 \%$ and $+20 \%$ or more is depicted by light gray, dark gray, and black boxes, respectively. A total of 6 patients in the P group did not undergo CT examination 1 month after the treatment and died of BSC policy. $\mathrm{CT}$, computed tomography; BSC, best supportive care.

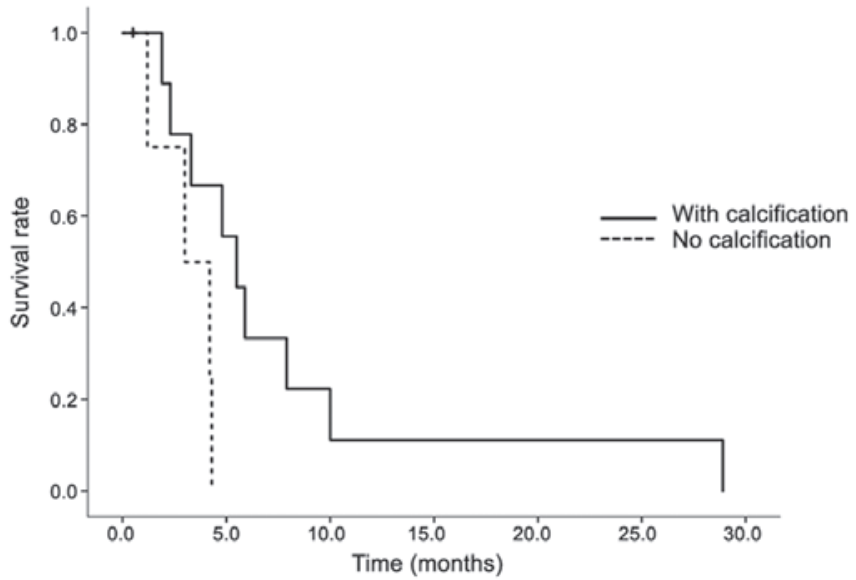

Figure 5. OS curves of patients with or without calcification in the primary lesion of ATC. Kaplan-Meier estimator (SPSS software)-derived survival curves indicate a median OS duration of 3.15 months in patients without calcification and 3.00 months in patients with calcification. There was no significant difference in OS curves between the two groups of patients $(\mathrm{P}=0.312)$. OS, overall survival; ATC, anaplastic thyroid cancer.

Antitumor effect. Changes in tumor diameter after first 4 weeks of treatment are shown in Fig. 4. A reduction in tumor diameter by $\geq 30 \%$ [clinical partial response (cPR)] was observed in 5 patients $(31.3 \%)$ in the L group and none in the $\mathrm{P}$ group. A total of 6 patients in the $\mathrm{P}$ group did not undergo a CT scan 1 month after the treatment and died of BSC policy. In the L group, the tumor reduction effect of lenvatinib was observed at either the full or low dose. In addition, treatment-related hypothyroidism occurred during the course of lenvatinib and PTX treatment; as a result, 14 patients $(87.5 \%)$ in the L group and 4 patients (25.0\%) in the $\mathrm{P}$ group required thyroid hormone replacement therapy. When the treatment period exceeded 3 months, particularly in the L group, the thyroid function in patients was severely compromised; thus, these patients required thyroid hormone replacement therapy. Although none of the patients in the present study had undergone total thyroidectomy for volume reduction, tumor necrosis by lenvatinib, also affecting normal thyroid gland tissue, caused hypothyroidism.

Difference by calcification pattern. Regarding tumor characteristics, 11 patients had tumors displaying an eggshell calcification pattern, 8 had lumpy calcified tumors, and 9 had non-calcified tumors. In the remaining 4 patients, calcification was found out of the tumor and its association with the tumor was unknown; therefore, it was excluded from this analysis. Comparing the treatment results of lenvatinib with and without calcification, the median OS was 5.5 months for patients with calcification and 3.0 months for those without calcification. The difference in the OS curves was statistically significant ( $\mathrm{P}=0.0473$; Fig. 5).

\section{Discussion}

The genetic analysis of ATC (12) demonstrated that some ATCs evolve from PTCs, FTCs and aggressive DTCs; the remaining cases are de novo carcinomas. An interesting previous study demonstrated that PTC coexisted with ATC and harbored the same BRAF mutation (13). Moreover, previous reports hypothesized that DTC is a pre-existing condition in the majority of ATC cases $(9,10)$. In fact, it is not uncommon to observe the coexistence of DTC in ATC pathological tissues $(14,15)$. In the present study, a co-existing PTC in the biopsied specimen was detected in 3 patients, and their CT images displayed calcification. The co-existence of DTCs in ATC surgical specimens is occasionally observed, but it is difficult to prove pre-existing DTC. It is desirable to determine the presence of DTC prior to the development of ATC. In the present study, CT examination at the time of diagnosis revealed calcification in the primary lesion, which may have resulted from a thyroid disease, such as DTC or multinodular goiter. Few studies have reported image findings for ATC. Takashima et al reported that 58\% of ATC patients had dense calcifications (16). Although this incidence was lower compared to the $67.9 \%$ in the present study, over half ATCs displayed calcifications. Thus, it may be hypothesized 
that pre-existing diseases may result in calcification in ATC. It was suggested that differentiation may affect lenvatinib efficacy, and the treatment results of lenvatinib were superior in patients who had calcifications in the primary lesion. In order to avoid treatment-related deaths due to fistula formation in all patients, discontinuation and dose reduction must be considered before the condition becomes life-threatening.

In a phase II study, the use of lenvatinib in 17 patients with ATC was reported $(5,6)$. The median progression-free survival was 7.4 months and the median OS was 10.6 months. The majority of the patients had been pretreated prior to lenvatinib treatment: 14 patients underwent surgery, 7 patients underwent chemotherapy, and 9 patients received external irradiation. The present study only compared patients receiving lenvatinib with those receiving palliative treatment for stage IVC ATC. Following lenvatinib treatment, 13 patients exhibited a reduction in tumor size, of whom 5 (31.3\%) achieved a cPR; 1 patient displayed an increase in tumor size after 1 month and 2 did not display any changes. Although this treatment response does not appear to be a bad result, we believe that extensive tumor necrosis, AE-related mortality and treatment discontinuation caused by formation of tumor-skin fistula may severely compromise treatment outcomes. It is crucial that a median 2.2-month survival benefit was obtained with lenvatinib compared with palliative therapy. In addition, it was reported that an initial low dose of lenvatinib $(10,14$ and $20 \mathrm{mg})$ did not alter the results of DTC (17). Recently, ATC has been treated with low-dose lenvatinib, depending on the patient's condition. In ATC, as the tumor is close to the skin, treatment should be initiated as soon as possible after confirmation of diagnosis using core needle biopsy, as a fistula may form where the tumor is exposed at the biopsy site. As ATC progresses rapidly, prompt initiation of treatment is required; moreover, it is necessary to arrest tumor progression and administer a proper dose to avoid deterioration of the fistula. This is also to avoid treatment-related deaths due to profuse bleeding from large vessels located close to the fistula. In the present study, co-existing PTC was detected in 3 patients, who also displayed calcifications on the CT scan. It has been reported that DTC coexisted in $35 \%$ of patients with ATC (2) and that ATC coexists not only with DTC, but also with thyroid diseases, such as nodular goiter (3). The calcification patterns identified in our study displayed the typical characteristics of PTC, and 3 patients had coexisting PTC, which was detected by core needle biopsy; therefore, transformation of PTC to ATC was suggested.

In addition to than lenvatinib, BRAF inhibitors serve as effective therapeutic agents for type I ATC with BRAF mutation (18). Programmed death-1 checkpoint blockers may be also efficacious in patients with aggressive forms of thyroid cancer (19). However, these agents are not approved for use in Japan. The combination of PLX4720 and dasatinib induced apoptosis, increased immune cell infiltration and reduced tumor volume in a preclinical model of ATC, and may be expected to be approved as treatment for patients with ATC in the future (20). The results of the present study may serve as a basis for progress in ATC treatment, including combination treatment with lenvatinib.

In conclusion, the $\mathrm{L}$ group demonstrated a median survival benefit of 2.2 months compared with the P group in stage IVC
ATC. Although cPR was confirmed in 5 patients who received lenvatinib, 2 patients died. These results suggest that an appropriate lenvatinib dose reduction is necessary.

\section{Acknowledgements}

Not applicable.

\section{Funding}

No funding was received.

\section{Availability of data and materials}

The datasets used and/or analyzed during the present study are available from the corresponding author on reasonable request.

\section{Authors' contributions}

HI and ST designed the study. NS and DM performed analysis and data interpretation, particularly statistical analysis. HN and KM contributed to data acquisition. All authors have read and approved the final version of the manuscript.

\section{Ethics approval and consent to participate}

The Chemotherapy Committee of Kanagawa Cancer Center (Yokohama, Japan), approved this regimen of lenvatinib for use in patients with ATC. The cancer board of our Kanagawa Cancer Center also approved lenvatinib treatment, including surgery, for patients with ATC. All patients provided a comprehensive consent form stating that personal data may be used for academic presentation or paper presentation, while ensuring complete anonymity, prior to receiving the treatment.

\section{Patient consent for publication}

Not applicable.

\section{Competing interests}

All the authors declare that they have no competing financial interests. An abstract (submission ID \#34) covering this article was presented at the WCTC meeting held in Rome, Italy, on June 20-22, 2019.

\section{Authors' information}

The first author HI is an endocrine surgeon working at the Kanagawa Cancer Center and has extensive experience on several surgeries for advanced thyroid cancer, as well as ATC treatment.

\section{References}

1. Oh EM, Lee KE, Kwon H, Kim EY, Bae DS and Youn YK: Analysis of patients with anaplastic thyroid cancer expected to have curative surgery. J Korean Surg Soc 83: 123-129, 2012.

2. Shimaoka K, Schoenfeld DA, DeWys WD, Creech RH and DeConti R: A randomized trial of doxorubicin versus doxorubicin plus cisplatin in patients with advanced thyroid carcinoma. Cancer 56: 2155-2160, 1985. 
3. Mooney CJ, Nagaiah G, Fu P, Wasman JK, Cooney MM, Savvides PS, Bokar JA, Dowlati A, Wang D, Agarwala SS, et al: A phase II trial of fosbretabulin in advanced anaplastic thyroid carcinoma and correlation of baseline serum-soluble intracellular adhesion molecule-1 with outcome. Thyroid 19: 233-240, 2009.

4. Sosa JA, Elisei R, Jarzab B, Balkissoon J, Lu SP, Bal C, Marur S, Gramza A, Yosef RB, Gitlitz B, et al: Randomized safety and efficacy study of fosbretabulin with paclitaxel/carboplatin against anaplastic thyroid carcinoma. Thyroid 24: 232-240, 2014

5. Bisof V, Rakusic Z and Despot M: Treatment of patients with anaplastic thyroid cancer during the last 20 years: Whether any progress has been made? Eur Arch Otorhinolaryngol 272: $1553-1567,2015$.

6. Smallridge RC, Ain KB, Asa SL, Bible KC, Brierley JD, Burman KD, Kebebew E, Lee NY, Nikiforov YE, Rosenthal MS, et al: American thyroid association guidelines for management of patients with anaplastic thyroid cancer. Thyroid 22: 1104-1139, 2012.

7. Iwasaki H, Yamazaki H, Suganuma N, Nakayama H, Toda S and Masudo K: Role of tyrosine kinase inhibitor thyrapy in anaplastic thyroid cancer. Int J Recent Adv Multidisciplinary Res 05: 4270-4274, 2018

8. Iwasaki H, Yamazaki H, Takasaki H, Suganuma N, Nakayama H, Toda S and Masudo K: Lenvatinib as a novel treatment for anaplastic thyroid cancer: A retrospective study. Oncol Lett 16 : 7271-7277, 2018.

9. Venkatesh YS, Ordonez NG, Schultz PN, Hickey RC, Goepfert H and Samaan NA: Anaplastic carcinoma of the thyroid. A clinicopathologic study of 121 cases. Cancer 66: 321-330, 1990.

10. Kebebew E, Greenspan FS, Clark OH, Woeber KA and McMillan A: Anaplastic thyroid carcinoma. Treatment outcome and prognostic factors. Cancer 103: 1330-1335, 2005.

11. Onoda N, Sugino K, Higashiyama T, Kammori M, Toda K, Ito K, Yoshida A, Suganuma N, Nakashima N, Suzuki S, et al: The safety and efficacy of weekly paclitaxel administration for anaplastic thyroid cancer patients: A nationwide prospective study. Thyroid 26: 1293-1299, 2016

12. Pozdeyev N, Gay LM, Sokol ES, Hartmaier R, Deaver KE, Davis S, French JD, Borre PV, LaBarbera DV, Tan AC, et al: Genetic analysis of 779 advanced differentiated and anaplastic thyroid cancers. Clin Cancer Res 24: 3059-3068, 2018.
13. Begum S, Rosenbaum E, Henrique R, Cohen Y, Sidransky D and Westra WH: BRAF mutations in anaplastic thyroid carcinoma: Implications for tumor origin, diagnosis and treatment. Mod Pathol 17: 1359-1363, 2004.

14. McIver B, Hay ID, Giuffrida DF, Dvorak CE, Grant CS Thompson GB, van Heerden JA and Goellner JR: Anaplastic thyroid carcinoma: A 50-year experience at a single institution. Surgery 130: 1028-1034, 2001.

15. Pacheco-Ojeda LA, Martinez AL and Alvarez M: Anaplastic thyroid carcinoma in ecuador: Analysis of prognostic factors. Int Surg 86: 117-121, 2001.

16. Takashima S, Morimoto S, Ikezoe J, Takai S, Kobayashi T, Koyama H, Nishiyama K and Kozuka T: CT evaluation of anaplastic thyroid carcinoma. AJR Am J Roentgenol 154: 1079-1085, 1990

17. Yamazaki H, Iwasaki H, Takasaki H, Suganuma N, Sakai R, Masudo K, Nakayama H, Rino Y and Masuda M: Efficacy and tolerability of initial low-dose lenvatinib to treat differentiated thyroid cancer. Medicine (Baltimore) 98: e14774, 2019.

18. Subbiah V, Kreitman RJ, Wainberg ZA, Cho JY, Schellens JHM, Soria JC, Wen PY, Zielinski C, Cabanillas ME, Urbanowitz G, et al: Dabrafenib and trametinib treatment in patients with locally advanced or metastatic BRAF V600-mutant anaplastic thyroid cancer. J Clin Oncol 36: 7-13, 2018.

19. Bastman JJ, Serracino HS, Zhu Y, Koenig MR, Mateescu V, Sams SB, Davies KD, Raeburn CD, McIntyre RC Jr, Haugen BR, et al: Tumor-Infiltrating $\mathrm{T}$ cells and the PD-1 checkpoint pathway in advanced differentiated and anaplastic thyroid cancer. J Clin Endocrinol Metab 101: 2863-2873, 2016.

20. Vanden Borre P, Gunda V, McFadden DG, Sadow PM, Varmeh S, Bernasconi M, Parangi S, et al: Combined BRAF(V600E)-and SRC-inhibition induces apoptosis, evokes an immune response and reduces tumor growth in an immunocompetent orthotopic mouse model of anaplastic thyroid cancer. Oncotarget 5: 39964010,2014

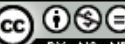

This work is licensed under a Creative Commons Attribution-NonCommercial-NoDerivatives 4.0 International (CC BY-NC-ND 4.0) License. 